\title{
Activating natural product synthesis using CRISPR interference and activation systems in Streptomyces.
}

Andrea Ameruoso ${ }^{1}$, Maria Claudia Villegas Kcam ${ }^{1}$, Katherine Piper Cohen ${ }^{1}$ and James Chappell ${ }^{1,2 *}$

1 - Department of BioSciences, Rice University, 6100 Main Street, MS 140, Houston, TX 77005, USA.

2 - Department of Bioengineering, Rice University, 6100 Main Street, MS 142, Houston, TX 77005, USA.

${ }^{*}$ Corresponding author: jc125@rice.edu

\section{ABSTRACT}

The rise of antibiotic-resistant bacteria represents a major threat to global health, creating an urgent need to discover new antibiotics. Natural products derived from the genus Streptomyces represent a rich and diverse repertoire of chemical molecules from which new antibiotics are likely to be found. However, a major challenge is that the biosynthetic gene clusters (BGCs) responsible for natural product synthesis are often poorly expressed under laboratory culturing conditions, thus preventing isolation and screening of novel chemicals. To address this, we describe a novel approach to activate silent BGCs through rewiring endogenous regulation using synthetic gene regulators based upon CRISPR-Cas. First, we create CRISPR interference (CRISPRi) and CRISPR activation (CRISPRa) systems that allow for highly programmable and effective gene repression and activation in Streptomyces. We then harness these tools to activate a silent BGC through perturbing its endogenous regulatory network. Together, this work advances the synthetic regulatory toolbox for Streptomyces and facilitates the programmable activation of silent BGCs for novel chemical discovery.

\section{INTRODUCTION}

Bacterial resistance to antimicrobial agents is globally recognized as one of the major challenges facing public health ${ }^{1,2}$. Addressing the antibiotic resistance crisis requires a multipronged approach, including the discovery of new antibiotics for which resistance has not been reported ${ }^{3}$. However, over the last decades, there has been a sharp decline 
in the discovery of new antibiotics, and it is widely recognized that innovative approaches for antibiotic discovery are required ${ }^{4}$.

Natural products derived from Streptomyces species are a likely major source of new antibiotics ${ }^{5}$. Microbial natural products are encoded by convoluted genomic regions called Biosynthetic Gene Clusters (BGCs). BGCs typically consist of dozens of genes that are co-localized at a single portion of a bacterial genome and encode components for natural product synthesis, transport, regulation, and resistance ${ }^{6}$. While natural products derived from Streptomyces species were once thought to be exhausted, advances in bioinformatic tools and genome sequencing have revealed that BGCs are far more abundant than previously thought, averaging 39 per genome ${ }^{6}$. Additionally, greater diversity is likely to exist: evidence indicates that the same species can vary tremendously in the BGCs they carry, due to high-rates of horizontal gene transfer and formation of novel clusters through duplication and rearrangement events ${ }^{6}$. Furthermore, new natural products are likely to come from exploring Streptomyces species beyond traditionally mined soil microbiomes. For example, Streptomyces have been found within hostassociated microbiomes of higher-order eukaryotes such as humans ${ }^{7}$, marine organisms ${ }^{8}$ and insects ${ }^{9}$, which are likely to contain different chemical diversity due to distinct evolutionary trajectories and environmental ecologies ${ }^{10}$.

While a rich repertoire of BGCs exist, the vast majority remain uncharacterized ${ }^{11}$. A key reason for this lack of extensive characterization is that the majority of BGCs are expressed poorly or not at all when grown under laboratory conditions ${ }^{12}$. This is due to stringent expression control imposed by networks of gene regulators located within the BGC, called cluster-situated regulators (CSRs), and/or global regulators encoded at distinct genomic loci. These networks ensure that BGCs are only expressed under certain environmental stimuli ${ }^{13}$. Identifying these stimuli to induce BGC expression in the laboratory requires screening of large parameter spaces ${ }^{10}$. Additionally, it is hard to activate specific and individual BGCs using this strategy ${ }^{5}$. Taken together, there is strong motivation to find new synthetic routes to predictably and precisely activate silent BGCs.

Engineering of CRISPR-Cas systems has led to a powerful suite of trans-acting gene regulatory tools able to precisely program gene expression in bacteria ${ }^{14-16}$. For example, CRISPR interference (CRISPRi) leverages a catalytically inactive version of the 
Streptococcus pyogenes Cas9 (dead Cas9, or dCas9) and a single guide RNA (sgRNA) to target DNA to sterically block transcription initiation and elongation ${ }^{17,18}$. In addition to gene repression, CRISPR activation (CRISPRa) systems able to turn on transcription of a target gene have also been created $^{18-23}$. CRISPR activation is achieved by recruiting protein activation domains (ADs) to the dCas9 complex that stimulate transcription when localized upstream of promoter elements. The power of these regulatory tools lies in the facile synthesis and flexibility of sgRNAs, which can be designed to target any DNA sequence that is proximal to a three-nucleotide protospacer adjacent motif (PAM). Additionally, because these systems act in trans, they can be encoded on easilytransferable plasmids and provide a route to perturb the expression of chromosomeencoded genes without the need for chromosome engineering that can be timeconsuming and arduous. Taken together, CRISPRi and CRISPRa provide a strategy to rapidly and precisely perturb the expression of endogenous genes.

We posit that CRISPRi and CRISPRa can provide a novel approach to activate BGC expression through perturbing and rewiring the underlying regulatory gene networks. For example, CRISPRa could be used to directly activate BGCs or to induce overexpression of endogenous transcription activators controlling BGC expression. Likewise, CRISPRi could be used to relieve BGC repression exerted by endogenous regulators. Importantly, the CRISPRi regulatory mechanism is highly portable, and CRISPRi tools have been developed for Streptomyces species. While these tools have been applied to turn off BGC expression ${ }^{24-26}$ and redirect metabolic flux from primary to secondary metabolism ${ }^{27}$, their application to activate silent BGC has yet to be explored. As for CRISPRa, its development has proven especially challenging in bacteria, and the majority of efforts have focused on optimizing these technologies for the model species E. coli ${ }^{18-23}$. Crucially, CRISPRa systems have not been demonstrated in non-model species with high biosynthetic potential, such as Streptomyces, or applied to activate natural product synthesis.

To address this, here we applied CRISPRi and CRISPRa to the activation of BGCs in Streptomyces venezuelae (S. venezuelae). We first characterized the performance of CRISPRi by exploring key parameters with a fluorescent reporter. Next, we established a functional CRISPRa system and explored its design rules. Finally, we demonstrated the 
applicability of both CRISPR tools for the activation of a silent BGC. Overall, our work introduces a facile and predictable strategy for the activation of silent BGCs within Streptomyces.

\section{RESULTS}

\section{Creating a CRISPRi platform for programmable transcription repression in}

Streptomyces venezuelae. As a starting point, our goal was to establish a CRISPRi system optimized for performance in $S$. venezuelae, which is an increasingly important biotechnological strain rich in silent $\mathrm{BGCs}^{28}$. The most established CRISPRi system leverages a dCas9 derived from $S$. pyogenes and a synthetic sgRNA ${ }^{17}$. To achieve repression, the sgRNA can be designed to bind the target gene's promoter, preventing transcription initiation, and thus turning off the expression of the gene (Figure 1a). Alternatively, this complex can be targeted to the coding sequence of a gene to sterically block transcription elongation (Figure 1b). To initially characterize CRISPRi in $S$. venezuelae, we constructed plasmids that express a codon-optimized dCas 9 and a sgRNA from the constitutive promoters $\operatorname{rpsL}(X C)$ and gapdh(EL), respectively. To measure transcription repression, we created a reporter strain in which a constitutive mCherry expression cassette, under the control of the KasOp* promoter, was integrated into the $\Phi C 31$ site of $S$. venezuelae as previously described ${ }^{29}$. We designed and cloned a corresponding sgRNA to target a PAM located at $11 \mathrm{bp}$ on the non-template strand within the mCherry reporter gene. Additionally, a plasmid containing only the antibiotic resistance cassette was constructed to be used as a no-CRISPRi control. We conjugated these plasmids into the $S$. venezuelae reporter strain and measured mCherry fluorescence (587 nm excitation and $610 \mathrm{~nm}$ emission) and optical density at $600 \mathrm{~nm}$ for each culture. From these experiments we observed that compared to the no-CRISPRi control, CRISPRi produced significant repression of fluorescence (Figure 1b).

While successful, we next sought to optimize the performance of CRISPRi by tuning the expression of its components. To do this, we first constructed a library of natural and synthetic constitutive promoters and characterized their expression strength through mCherry fluorescence assays (Supplementary figure 1). Subsequently, we decided to replace the gapdh(EL) promoter driving the expression of the sgRNA with the SP43 
promoter $^{30}$. SP43 is not only stronger than gapdh(EL) but has an annotated transcription start site (TSS) that allows for precise expression of the sgRNA without amending additional promoter-encoded sequences onto this transcript. When comparing the two conditions in mCherry fluorescence assays, we observed greater reduction in fluorescence in the presence of SP43 than in the presence of gapdh(EL), indicating stronger CRISPRi repression (Figure 1b). Next, we investigated the effect of dCas9 expression by replacing the original $\operatorname{rspL}(\mathrm{XC})$ promoter with a weaker (SP1) and stronger (SP30) promoter (Supplementary figure 1), while keeping the sgRNA under the control of SP43. We observed little difference in the level of transcription repression in response to different dCas9 expression levels (Figure 1c). Interestingly, while performing these experiments we observed a significant decrease in fluorescence in two cases when dCas9 was present: in absence of a sgRNA or in the presence of a non-targeting sgRNA (i.e. a sgRNA designed to target a DNA sequence absent in Streptomyces) (Supplementary figure 2). In contrast, inclusion of dCas9 alongside a sgRNA targeting a non-coding region in the genome but not mCherry, resulted in no decrease in fluorescence when compared to a no-CRISPRi control (Supplementary figure 2). Characterizing the growth rate of these control conditions uncovered significant growth defects when dCas9 is present either with a non-targeting sgRNA or without a sgRNA (Supplementary figure 3). While the exact cause of this effect is unknown, we reason they are likely due to nonspecific binding of dCas9 to DNA ${ }^{31}$ that appears to be particularly detrimental for Streptomyces, potentially due to the high frequency of PAMs within its GC rich genome ${ }^{32}$.

Having optimized a CRISPRi platform for S. venezuelae we next sought to gain deeper insight into the design rules. Specifically, we were interested in understanding how the strand (i.e., non-template or template) and position of CRISPRi targeting within the coding sequence affected the level of transcription repression. To test this, we designed and cloned a series of sgRNAs targeting PAMs in the non-template and template strand of mCherry located at 11, 123, 230, 531, 623, bp and 29, 132, 242, 560, $647 \mathrm{bp}$ respectively. From these experiments we saw significant repression when CRISPRi is targeted to the 5' end of the coding sequence on the non-template strand and negligible repression when targeting either the template strand or downstream regions of 
the non-template strand (Figure 1d). This is consistent with previous results of CRISPRi in other microbes ${ }^{33}$.

Creating a CRISPRa platform for transcription activation in S. venezuelae. While CRISPRa systems have been developed for $E$. coli, their application in other bacteria is lacking. For example, to date there has only been a single demonstration in a grampositive species, Bacillus subtilis ${ }^{34}$. To address this, our goal was to establish a CRISPRa platform in Streptomyces for the first time. The most established CRISPRa design motif relies upon translationally fusing dCas9 to a protein-based activation domain (AD) that, when localized close to promoter elements, activates transcription through recruitment of the RNAP or stabilization of the RNAP initiation complex (Figure 2a). Variations of this design include use of different ADs, linker sequences, and recruitment strategies ${ }^{18,19,21,22}$.

To create a CRISPRa system, we first sought to identify a functional AD for $S$. venezuelae. Specifically, we decided to investigate the $\omega$ subunit and $\mathrm{N}$ terminal domain of the $\alpha$ subunit ( $\alpha$ NTD) of RNAP as ADs. The $\omega$ subunit is a nonessential component of RNAP and plays a role in structurally stabilizing the holoenzyme, functionalities that can activate transcription when localized to promoter elements ${ }^{35}$. The $\omega$ subunit was the first $\mathrm{AD}$ used in a bacterial CRISPRa system in $E$. coli ${ }^{18}$, and has been adapted for use in other species ${ }^{34}$. The $\alpha$ NTD is responsible for initiating RNAP assembly and has recently been demonstrated as a robust $A D^{22}$. In addition to these $A D s$, we also considered a transcription-factor-based AD. Specifically, we investigated the RNA polymerase binding protein $A(R b p A)$, a transcription activator unique to Actinobacteria ${ }^{36}$. RbpA activates transcription through a combination of stabilizing RNAP-promoter open complex and recruiting the principal $\sigma$ factor, functionalities that we hypothesized would allow $\mathrm{RbpA}$ to serve as an AD. Plasmids were constructed in which the aNTD, $\omega$ and RbpA ADs derived from $S$. venezuelae were translationally fused to the $C$ terminus of dCas9. For the fusion, we employed a synthetic XTEN linker (SGSETPGTSESATPES) that has seen broad utility for creating chimeric fusions with Cas proteins ${ }^{22,37,38}$. To evaluate the performance of CRISPRa, we constructed a reporter cassette where an mCherry gene was placed under the control of the SP10 synthetic promoter, which we then integrated at the ФC31 site of S. venezuelae. We then designed sgRNAs to direct CRISPRa to sites upstream of 
the SP10 promoter. Specifically, we targeted PAM sites located at $83 \mathrm{bp}$ upstream of the promoter's transcription start site (TSS) on the non-template strand and at 82 bp upstream of TSS on the template strand. As a control for non-specific activation, for each CRISPRa design, we used an off-target sgRNA binding to a non-coding region in the genome and a no-CRISPRa condition. From these measurements we observed significant activation of mCherry expression when targeting the non-template strand with the aNTD AD (Figure 2b). No activation was observed from the RbpA or the $\omega$ subunit $A D$, which could be due to competition with the endogenous $\omega$ subunit for the RNAP, as has been observed in other species ${ }^{18,19,21,22}$. Overall, these results show we can create a functional CRISPRa system for Streptomyces for the first time using the aNTD AD.

Distance dependent activation of CRISPRa in S. venezuelae. An intriguing design constraint of CRISPRa systems in E. coli are periodical activation patterns in which activation is only observed when the system is targeted to PAMs within a 2-4 bp window that repeat every $10-11$ bp from the promoter's TSS ${ }^{20,22}$. It has been proposed that these patterns are due to the requirement for ADs to be localized on specific faces of the DNA helix relative to the targeted promoter's TSS ${ }^{20,22}$. These activation patterns have been observed for different ADs, and are independent of the dCas9-AD linker sequence and recruitment strategies being used (i.e., recruitment through binding sgRNA or dCas9) ${ }^{20,22}$. To determine if periodicity in activation is observed for CRISPRa in Streptomyces, we introduced PAM sites on the non-template strand at 73, 78, 83, 88, 93 bp upstream of the mCherry promoter's TSS that was integrated within the $S$. venezuelae genome (Figure 2c). Corresponding sgRNAs were designed and transcription activation was measured via $\mathrm{mCherry}$ fluorescence. Importantly, as has been reported in $E$. coli, we observed strong activation patterns that appear to repeat with a 10 bp periodicity (Figure 2c, Supplementary Figure 4). Specifically, we see more than 20 -fold activation when targeting PAMs located at 73, 83, and 93 bp from the TSS, and no activation when targeting PAMs at 78 and $88 \mathrm{bp}$. This demonstrates that CRISPRa in Streptomyces exhibits periodical activation patterns that need to be considered when deploying this new regulatory tool. 
Activating a silent BGC using CRISPRi and CRISPRa regulators. We next sought to demonstrate the utility of CRISPR-Cas regulatory tools for activating silent BGCs through perturbation and rewiring of endogenous regulation. For this, we decided to focus on the S. venezuelae jadomycin B (jdB) cluster, which encodes a type II polyketide synthase biosynthetic pathway. This cluster spans $\sim 28 \mathrm{~kb}$ and includes 31 genes: 23 biosynthetic genes, 7 regulatory genes and 1 transporter gene (Figure 3a). The expression of the jdB cluster is under the control of a highly complicated gene regulatory network involving numerous regulators including JadR1, JadR2, JadR3, JadR* , and JadW1-W3. While our understanding of the regulators and network is likely incomplete, two of the most prominent regulators are JadR1 and JadR2 (Figure 3b). JadR1 is the main activator that directly turns on the expression of the jadJ-V operon in the presence of low levels of jdB and is essential for jdB production. However, at high levels of jdB, jadR1 represses its own promoter ${ }^{39}$. JadR2, a pseudo $y$-butyrolactone (GBL) receptor, is the main cluster repressor as it directly shuts off jadR1 expression, thus indirectly repressing BGC activation $^{40}$. The net result of this regulation is that under standard laboratory culturing conditions the jdB cluster is not expressed. Interestingly, in the presence of environmental stressors such as ethanol shock, heat shock, and phage infection, jadR2 repression can be relieved and jdB synthesis induced. Inspired by this, we reasoned that CRISPRi could be used to synthetically knock down the expression of JadR2, relieving JadR1 repression and thus activating jdB synthesis (Figure 3c). To test this, we created a JadR2-repressing CRISPRi plasmid and conjugated it into wild-type S. venezuelae cells. A no-CRISPRi condition, containing an empty plasmid, was conjugated in parallel as a negative control. After growing and fermenting the exconjugants, solvent extraction was performed on the mycelia, and liquid chromatography-mass spectrometry (LC/MS) analysis was performed on the crude extracts. From these measurements, we observed production of jadomycin $\mathrm{B}\left(\mathrm{m} / \mathrm{z} 550.2059,[\mathrm{M}+\mathrm{H}]^{+}\right)$in the presence of CRISPRi, but not in the no-CRISPRi control (Figure 3c, Supplementary Fig 5). This demonstrated that CRISPRi can be used as a tool to activate silent BGCs by relieving the regulation of endogenous repressors.

Next, we sought to demonstrate that CRISPRa could also be used to induce activation of the jdB cluster. Specifically, our idea was to use CRISPRa to synthetically induce expression of the main biosynthetic operon (jadJ-V), in essence, rewiring the 
native regulatory network (Figure $3 \mathrm{~d}$ ). To this end, we conjugated a jadJV-activating CRISPRa plasmid into wild-type S. venezuelae cells. Subsequently, we tested the ability of CRISPRa to induce production of jdB by performing LC/MS analysis on the crude extracts obtained from fermentation, as described above. We detected the production of $\mathrm{jdB}$ in the presence of CRISPRa, but not in the control, demonstrating the ability of CRISPRa to activate silent BGCs through direct activation of key biosynthetic genes (Figure 3d, Supplementary Fig 6).

Taken together, these data show that our CRISPR regulatory tools can effectively be used to synthetically perturb and rewire the endogenous regulation of the jdB BGC and in doing so, activate the expression of this silent BGC to induce natural product synthesis.

\section{DISCUSSION}

In our work, we establish two CRISPR-Cas systems for gene expression control in Streptomyces, and successfully use them to activate a silent BGC. Specifically, we optimize and resolve the design rules for our CRISPRi system in Streptomyces and demonstrate, for the first time, its ability to activate a silent BGC through relieving the repression of endogenous regulators. In addition, we provide the first example of a CRISPRa system for Streptomyces, and demonstrate the ability of this system to directly activate BGC expression through targeting 'silent' promoter elements. Collectively, this work provides an expanded toolbox for gene expression control in Streptomyces and more broadly, demonstrates a new framework for using CRISPR-Cas regulators for natural product discovery.

Our work advances the available molecular tools for engineering Streptomyces, which is an increasingly important genus for drug discovery and biomanufacturing. Specifically, we created an optimized CRISPRi system for S. venezuelae and resolved design rules. Interestingly, we observed robust repression from CRISPRi and design rules that are consistent with previous studies in bacteria ${ }^{33}$, adding further evidence that CRISPRi is a highly portable regulatory mechanism across the bacteria domain of life. Additionally, we created the first example of CRISPRa for Streptomyces. Importantly, while CRISPRi has been demonstrated across diverse bacterial species, CRISPRa 
systems have largely been restricted to model Gram-negative bacteria (e.g., E. coli), with only a single demonstration in a gram-positive bacterium, B. subtilis ${ }^{34}$. Part of the challenge of CRISPRa is the identification of functional ADs for each host. Our results, along with other recent demonstrations in E.coli22, suggest that host-derived $\alpha$ NTD AD can serve as a potentially generalizable strategy to create CRISPRa systems for diverse bacterial species. We anticipate the creation of new CRISPR-Cas regulatory systems will advance both basic science investigations of Streptomyces and application-specific manipulations. For example, Streptomyces are increasingly being utilized for commercial production of natural products ${ }^{41}$. However, a major challenge are low titers of natural products that require optimization to ensure viable economics ${ }^{42}$. Our CRISPR-Cas tools provide a new capability to program endogenous gene expression to increase flux through desired pathways, while minimizing flux through competing pathways, as has been widely demonstrated in other microbes ${ }^{43-50}$.

Our work introduces a novel pathway-specific approach to activate silent BGCs that complements existing strategies. Previous pathway-specific methods have largely relied on refactoring BGCs directly on the host's genome or within heterologous hosts ${ }^{11}$. While successful, this approach can be arduous and challenging due to the complexity and size of BGCs. In contrast, CRISPR-Cas regulators provide a potentially more scalable framework. Libraries of sgRNAs are cheap and easy to synthesize, and regulators are easily-transferred into desired hosts through established and efficient conjugation methods. CRISPR-Cas regulators can also be used for pooled screening ${ }^{51}$, which we envisage could be used to multiplex different perturbations in a single experiment. More broadly, our work adds to a growing set of synthetic biology technologies for BGC activation ${ }^{11,52-54}$, which we anticipate can be synergistically combined in the future. For example, DNA-based transcription factor decoys have been utilized to relieve endogenous repression that could be used alongside our CRISPRi and CRISPRa technologies ${ }^{53}$.

While our CRISPR-Cas regulators represent a novel approach to activate BGCs, challenges remain. In particular, we observed that CRISPRa systems are only able to activate transcription when targeted to specific positions relative to the TSS. This periodical $10 \mathrm{bp}$ activation pattern is proposed to be due to the rotation of the DNA double 
helix ( $10.5 \mathrm{bp})$ and the requirement of the AD to be localized to specific faces of the DNA relative to the promoter 20,22 . While the likelihood of encountering an NGG PAM is high in Streptomyces spp. given their $\sim 70 \%$ GC content ${ }^{55}$, not all promoters will possess optimally positioned PAMs. Importantly, several strategies have been demonstrated in E.coli to overcome this limitation, which we anticipate could be applied to Streptomyces. For example, engineered dCas9 proteins with relaxed PAM requirements such $\mathrm{dxCas}(3.7)$ have been used for CRISPRa systems to allow activation from non-canonical $\mathrm{PAMs}^{20}$. Additionally, circularly permuted dCas9 proteins (cpdCas9) have also been used to create CRISPRa systems with distinct, non-overlapping activation patterns ${ }^{22}$. Finally, alternative CRISPR-Cas systems beyond type II have also been used to create CRISPRa systems with distinct regulatory properties ${ }^{56}$. Beyond the limitations of CRISPRa, we anticipate more work is required to understand the generalizability of our approach. For example, it remains to be elucidated if CRISPRa is capable of activating the diversity of different endogenous promoter elements. Finally, we anticipate deeper understanding of the regulatory networks controlling natural product synthesis will be required to precisely engineer the corresponding metabolic pathways.

In summary, this work expands the current synthetic biology tools to activate silent BGCs by leveraging CRISPR-Cas regulators to perturbate and rewire the underlying regulatory gene networks. While more work is still needed to expand and generalize the approach, our system demonstrates the applicability of CRISPRi and CRISPRa systems to activate BGCs in Streptomyces. This method only requires the design of a specific sgRNA and the straightforward transformation of a single plasmid, thus representing a simple approach to activate silent BGCs. We anticipate that this work will pave the way for the development of high-throughput technologies for the discovery of novel secondary metabolites on a large scale.

\section{METHODS}

Plasmid assembly. All plasmids used in this study can be found in Supplementary Table 1 with key sequences provided in Supplementary Tables 2-5. All plasmids were constructed with either Gibson assembly ${ }^{57}$, Golden Gate assembly ${ }^{58}$, or inverse PCR 
(iPCR). DNA manipulations were performed in E. coli strain NEB Turbo. Enzymes were obtained from New England Biolabs.

Strains and growth media. E. coli strains were grown in Luria Bertani (LB) broth, containing $50 \mu \mathrm{g} / \mathrm{ml}$ spectinomycin or $50 \mu \mathrm{g} / \mathrm{mL}$ apramycin as needed. 2,6-diaminopimelic acid was added to LB at a final concentration of $0.1 \mathrm{mM}$ for culturing $E$. coli strain WM602959. S. venezuelae ATCC 10712 was cultured in complete supplement medium (CSM) unless otherwise indicated. To prepare CSM, $30 \mathrm{~g}$ of tryptic soy broth, $1.2 \mathrm{~g}$ of Yeast Extract, and $1 \mathrm{~g}$ of $\mathrm{MgSO}_{4}$ were added to $1 \mathrm{~L}$ of water and autoclaved; filtersterilized D-(+)-glucose and D-(+)-maltose were then added at a final concentration of 28 $\mathrm{mM}$ and $12 \mathrm{mM}$, respectively. Conjugation involving WM6029 and S. venezuelae was conducted on AS-1 medium. AS-1 was prepared by adding $5 \mathrm{~g}$ of soluble starch, $2.5 \mathrm{~g}$ of $\mathrm{NaCl}, 1 \mathrm{~g}$ of yeast extract and $18 \mathrm{~g}$ of agar to $\mathrm{ddH}_{2} \mathrm{O}$ to a final volume of $1 \mathrm{~L}$ and then autoclaved. A filter-sterilized solution of alanine, arginine and asparagine was added to a final concentration of $0.02 \% \mathrm{w} / \mathrm{v}$ of each amino acid. Finally, an autoclaved $\mathrm{Na}_{2} \mathrm{SO}_{4}$ solution was added to a final concentration of $1 \%$. To prepare the MSM minimal medium for fermentation experiments, $\mathrm{MgSO}_{4}(0.04 \%$, w/v), MOPS $(0.377 \%$, w/v), salt solution $(0.9 \%, v / v)$, trace mineral solution $(0.45 \%, v / v)$, and $0.2 \% \mathrm{w} / \mathrm{v} \mathrm{FeSO}_{4} \cdot 7 \mathrm{H}_{2} \mathrm{O}$ solution $(0.45 \%, v / v)$ were added to $\mathrm{ddH}_{2} \mathrm{O}$ and the $\mathrm{pH}$ was adjusted to 7.5 . The salt solution was made by addition of $\mathrm{NaCl}(1 \%, w / v)$ and $\mathrm{CaCl} 2(1 \%, w / v)$ to $\mathrm{ddH}_{2} \mathrm{O}$. The trace mineral solution was made by addition of $\mathrm{ZnSO}_{4} \cdot 7 \mathrm{H}_{2} \mathrm{O}\left(0.088 \%\right.$, w/v), $\mathrm{CuSO}_{4} \cdot 5 \mathrm{H}_{2} \mathrm{O}(0.0039 \%$, w/v), $\mathrm{MnSO}_{4} \cdot 4 \mathrm{H}_{2} \mathrm{O}(0.00061 \%, w / v), \mathrm{H}_{3} \mathrm{BO}_{3}(0.00057 \%, w / v)$, and $\left(\mathrm{NH}_{4}\right) \mathrm{Mo}_{7} \mathrm{O}_{24} \cdot 4 \mathrm{H}_{2} \mathrm{O}$ $(0.00037 \%, w / v)$ to $d_{d H_{2}} \mathrm{O}$.

Reporter strains construction. Constructs containing different promoter-mCherry combinations were assembled as described above using plasmid JBEl16292, harboring the ФС31 integration system, as a backbone. These reporter plasmids were subsequently conjugated (see below) and integrated into S. venezuelae ATCC 10712, thus yielding the reporter strains. 
Interspecies conjugation. E. coli WM6029 cells were transformed with the plasmids to be conjugated. Colonies were picked into liquid LB media containing the appropriate antibiotic and $0.1 \mathrm{mM}$ DAP, and grown overnight at $37^{\circ} \mathrm{C}$. At the same time, $\mathrm{S}$. venezuelae mycelia were grown overnight in CSM. Liquid cultures were then pelleted and the medium was removed. Each WM6029 sample was resuspended in $500 \mu \mathrm{L}$ of fresh LB with no antibiotics, while $S$. venezuelae pellets were resuspended in $2 \mathrm{~mL}$ of fresh CSM. WM6029 and S. venezuelae were then mixed at a 1:1 ratio, and each co-culture was spotted on AS-1 plates supplemented with 0.1 mM DAP. After incubating for 16-20 hours at $30{ }^{\circ} \mathrm{C}$, the plates were flooded with solutions containing $500 \mu \mathrm{g}$ of nalidixic acid and $1 \mathrm{mg}$ of the appropriate antibiotic. Plates were then stored at $30{ }^{\circ} \mathrm{C}$ until the appearance of exconjugant colonies (3 to 6 days). Exconjugant colonies were streaked on fresh ISP-2 plates supplemented with either apramycin or spectinomycin at a final concentration of $50 \mu \mathrm{g} / \mathrm{mL}$.

Fluorescence measurements. S. venezuelae exconjugants were picked from ISP-2 plates and used to inoculate $5 \mathrm{~mL}$ of CSM supplemented with antibiotics as needed (apramycin or spectinomycin, final concentration $50 \mu \mathrm{g} / \mathrm{mL}$ ). These seed cultures were grown for 2-3 days at $30{ }^{\circ} \mathrm{C}$, then diluted to an optical density at $600 \mathrm{~nm}\left(\mathrm{OD}_{600}\right)$ of 0.01 in fresh CSM supplemented with antibiotics, and grown for 24 hours. After 24 hours, 25 $\mu \mathrm{L}$ of each culture were transferred to $75 \mu \mathrm{L}$ of fresh CSM media supplemented with antibiotics as needed inside a 96 well microplate (Costar). OD600 (OD) and fluorescence (FL) (excitation $587 \mathrm{~nm}$ and emission $610 \mathrm{~nm}$ ) were measured in a microplate reader (Tecan Infinite M1000 Pro). When performing CRISPRi and CRISPRa experiments, a S. venezuelae strain harboring a previously integrated mCherry reporter was used as recipient for conjugation. After conjugation, fluorescence measurement experiments were carried out as described above.

Fluorescence data analysis. In each fluorescence measurement experiment, OD 600 and FL values for each sample were corrected by subtracting the mean OD and FL values of a media blank. The ratio of $F L$ to $\mathrm{OD}_{600}(\mathrm{FL} / \mathrm{OD})$ was then calculated. Data are reported 
as mean FL/OD values for each condition, and error bars represent standard deviation (s.d.).

Growth curve experiments. S. venezuelae colonies were grown at $30^{\circ} \mathrm{C}$ with $250 \mathrm{rpm}$ shaking in $5 \mathrm{~mL}$ CSM supplemented with apramycin or spectinomycin as needed. Upon reaching high cell density ( $\sim 2$ days), cells were diluted in the same medium to an $\mathrm{OD}_{600}$ of 0.08 directly in a Costar 96 well microplate (final volume $100 \mu \mathrm{L}$ ). Cells were then grown in a microplate reader (Tecan Spark multimode plate reader) at $30^{\circ} \mathrm{C}$ with $90 \mathrm{rpm}$ shaking for 24 hours. $\mathrm{OD}_{600}$ measurements were automatically taken every 10 minutes. For data analysis, $\mathrm{OD}_{600}$ values of the media controls were averaged at each time point, and then subtracted from the individual values of each condition at each time point.

Distance-dependent CRISPRa experiments. To evaluate distance-dependent effects, sgRNAs were designed to target sequences proximal to PAMs positioned on the nontemplate strand at a distance of 73, 83, 93 bp upstream of the reporter promoter's TSS . Distances do not include either the PAM or the TSS. The same sgRNAs were used to target sequences upstream of a reporter that contained 5 additional nucleotides to extend the distance between the TSS and each PAM by 5 bp (thus creating 78 and 88 bp binding sites). Fluorescence measurements were performed as described above, and FL/OD for each sample were calculated. As each reporter had slightly different mCherry expression values, data are reported as fold activation. Fold activation was calculated by normalizing FL/OD values of each sample to the mean FL/OD values of the no-CRISPRa control of each reporter. Corresponding FL/OD values used for fold activation calculations are shown in Supplementary figure 4.

Fermentation for jadomycin B production. Fermentations were conducted under previously described conditions ${ }^{60,61}$. CRISPRi or CRISPRa plasmids carrying appropriate sgRNAs were conjugated into wild-type $S$. venezuelae, as described above. Exconjugants were then picked to inoculate $100 \mathrm{~mL}$ of CSM supplemented with apramycin, and grown to high density (usually 3-5 days) in $250 \mathrm{~mL}$ Erlenmeyer flasks at $30^{\circ} \mathrm{C}$. Cultures were then centrifuged, and the pellets washed twice in MSM to remove 
any trace of CSM. After resuspension in $6 \mathrm{~mL}$ of MSM, each sample was diluted in MSM supplemented with apramycin to an $\mathrm{OD}_{600}$ of 0.6 and a final volume of $50 \mathrm{~mL}$. Fermentation cultures were incubated at $30{ }^{\circ} \mathrm{C}$ with $250 \mathrm{rpm}$ shaking. After 72 hours, cultures were centrifuged and the pellets were stored at $-80^{\circ} \mathrm{C}$ for LC-MS analysis.

Extraction and LC-MS analysis. Pellets obtained from fermentations were extracted with an equal volume of acetone. Mixtures were transferred to an Erlenmeyer flask and shaken at room temperature for 30 minutes at $180 \mathrm{rpm}$. Acetone was then evaporated in a rotovap at $40^{\circ} \mathrm{C}$ and 200 mbar. Crude extracts were reconstituted in $5 \mathrm{~mL}$ of acetonitrile, and $500 \mu \mathrm{L}$ of the reconstituted extract was combined with $500 \mu \mathrm{L}$ of LC-MS grade water. LC-MS was carried out on an Agilent 6470B Triple Quadrupole Mass Spectrometer interface to an Agilent 1290 Infinity II HPLC system through a Jet Spray ESI source. The LC column was an Agilent $2.1 \mathrm{~mm}$ ID x $50 \mathrm{~mm}, 1.8 \mu \mathrm{m}$ SB-C18 column. Mobile phase A was water with $0.1 \%$ formic acid, and mobile phase $B$ was acetonitrile with $0.1 \%$ formic acid. LC flow rate was $0.4 \mathrm{ml} / \mathrm{min}$. Initial conditions were $10 \% \mathrm{~B}$ up to $95 \% \mathrm{~B}$ over three minutes. The column was flushed with $95 \%$ B from 3-6 minutes and returned to initial conditions from 6-6.5 minutes. The column was equilibrated at initial conditions from 6.5$9.5 \mathrm{~min}$.

Data analysis. Experiments were performed using at least three biological replicates per condition tested unless otherwise indicated. Data for figures $1 \mathrm{~b}, 1 \mathrm{c}, 1 \mathrm{~d}$, and $2 \mathrm{~b}$ are reported as bars showing mean, with error bars showing standard deviation. Individual samples are also shown as black circles. Jadomycin B production experiments were performed in biological duplicates. Data in figures $3 d$ and $3 e$ show extracted ion chromatograms of one representative biological sample for each condition. All remaining biological replicates for LC-MS experiments are shown in Supplementary figures 5-6.

Data availability. All source data for main figures were deposited in Rice University's Rice Digital Scholarship Archive (DOI: https://doi.org/10.25611/5SAC-CW45) 


\section{Acknowledgments}

The authors would like to acknowledge William Metcalf (University of Illinois at UrbanaChampaign) for providing the WM6029 E. coli strain. The authors would like to acknowledge Jay Keasling (UC Berkeley) for providing the genomic integration plasmid JBEl16292 used to construct the reporter strains. The authors acknowledge the support of the Shared Equipment Authority at Rice University. In particular, the authors would like to acknowledge Christopher Pennington for his assistance on LC-MS experiments. This material is based upon work supported by the Welch Foundation [C-1982-20190330 to J.C] and the Alfred P. Sloan Research Fellowship [FG-2018-10500 to J.C]. J.C. is an Alfred P. Sloan Research Fellow. The authors declare no competing financial interest.

\section{AUTHOR CONTRIBUTIONS}

A.A., M.C.V.K, K.P.C., and J.C. designed the study, A.A. performed experiments. All authors contributed to data analysis and preparation of the manuscript.

\section{REFERENCES}

1. Centers for Disease Control and Prevention (U.S.). Antibiotic resistance threats in the United States, 2019. https://stacks.cdc.gov/view/cdc/82532 doi:10.15620/cdc:82532.

2. World Health Organization. Antimicrobial resistance: global report on surveillance. (World Health Organization, 2014).

3. Aslam, B. et al. Antibiotic resistance: a rundown of a global crisis. Infect. Drug Resist. 11, 1645-1658 (2018).

4. Spellberg, B., Bartlett, J. G. \& Gilbert, D. N. The Future of Antibiotics and Resistance. N. Engl. J. Med. 368, 299-302 (2013).

5. Rutledge, P. J. \& Challis, G. L. Discovery of microbial natural products by activation of silent biosynthetic gene clusters. Nat. Rev. Microbiol. 13, 509-523 (2015). 
6. Belknap, K. C., Park, C. J., Barth, B. M. \& Andam, C. P. Genome mining of biosynthetic and chemotherapeutic gene clusters in Streptomyces bacteria. Sci. Rep. 10, 2003 (2020).

7. Bolourian, A. \& Mojtahedi, Z. Streptomyces, shared microbiome member of soil and gut, as 'old friends' against colon cancer. FEMS Microbiol. Ecol. 94, (2018).

8. Zhang, H., Zhang, W., Jin, Y., Jin, M. \& Yu, X. A comparative study on the phylogenetic diversity of culturable actinobacteria isolated from five marine sponge species. Antonie Van Leeuwenhoek 93, 241-248 (2008).

9. Chevrette, M. G. et al. The antimicrobial potential of Streptomyces from insect microbiomes. Nat. Commun. 10, 516 (2019).

10. van Bergeijk, D. A., Terlouw, B. R., Medema, M. H. \& van Wezel, G. P. Ecology and genomics of Actinobacteria: new concepts for natural product discovery. Nat. Rev. Microbiol. 18, 546-558 (2020).

11. Smanski, M. J. et al. Synthetic biology to access and expand nature's chemical diversity. Nat. Rev. Microbiol. 14, 135-149 (2016).

12. Chiang, Y.-M., Chang, S.-L., Oakley, B. R. \& Wang, C. C. Recent advances in awakening silent biosynthetic gene clusters and linking orphan clusters to natural products in microorganisms. Curr. Opin. Chem. Biol. 15, 137-143 (2011).

13. Liu, G., Chater, K. F., Chandra, G., Niu, G. \& Tan, H. Molecular regulation of antibiotic biosynthesis in streptomyces. Microbiol. Mol. Biol. Rev. MMBR 77, 112-43 (2013).

14. Adli, M. The CRISPR tool kit for genome editing and beyond. Nat. Commun. 9, 1911 (2018).

15. Ameruoso, A., Gambill, L., Liu, B., Villegas Kcam, M. C. \& Chappell, J. Brave new 'RNA' world-advances in RNA tools and their application for understanding and engineering biological systems. Curr. Opin. Syst. Biol. 14, 32-40 (2019).

16. Xu, X. \& Qi, L. S. A CRISPR-dCas Toolbox for Genetic Engineering and Synthetic Biology. J. Mol. Biol. 431, 34-47 (2019).

17. Qi, L. S. et al. Repurposing CRISPR as an RNA-guided platform for sequencespecific control of gene expression. Cell 152, 1173-83 (2013). 
18. Bikard, D. et al. Programmable repression and activation of bacterial gene expression using an engineered CRISPR-Cas system. Nucleic Acids Res. 41, 7429-7437 (2013).

19. Dong, C., Fontana, J., Patel, A., Carothers, J. M. \& Zalatan, J. G. Synthetic CRISPR-Cas gene activators for transcriptional reprogramming in bacteria. Nat. Commun. 9, 2489-2489 (2018).

20. Fontana, J. et al. Effective CRISPRa-mediated control of gene expression in bacteria must overcome strict target site requirements. Nat. Commun. 11, 1618 (2020).

21. Ho, H., Fang, J. R., Cheung, J. \& Wang, H. H. Programmable CRISPR-Cas transcriptional activation in bacteria. Mol. Syst. Biol. 16, e9427 (2020).

22. Villegas Kcam, M. C., Tsong, A. J. \& Chappell, J. Rational engineering of a modular bacterial CRISPR-Cas activation platform with expanded target range. Nucleic Acids Res. 49, 4793-4802 (2021).

23. Liu, Y., Wan, X. \& Wang, B. Engineered CRISPRa enables programmable eukaryote-like gene activation in bacteria. Nat. Commun. 10, 3693-3693 (2019).

24. Zhao, Y. et al. CRISPR/dCas9-Mediated Multiplex Gene Repression in Streptomyces. Biotechnol. J. 13, 1800121 (2018).

25. Tong, Y., Charusanti, P., Zhang, L., Weber, T. \& Lee, S. Y. CRISPR-Cas9 Based Engineering of Actinomycetal Genomes. ACS Synth. Biol. 4, 1020-1029 (2015).

26. Li, L. et al. CRISPR-Cpf1-Assisted Multiplex Genome Editing and Transcriptional Repression in Streptomyces. Appl. Environ. Microbiol. 84, e00827-18 (2018).

27. Tian, J. et al. Developing an endogenous quorum-sensing based CRISPRi circuit for autonomous and tunable dynamic regulation of multiple targets in Streptomyces. Nucleic Acids Res. 48, 8188-8202 (2020).

28. Chater, K. F. Recent advances in understanding Streptomyces. F1000Research 5, 2795-2795 (2016).

29. Phelan, R. M. et al. Development of next generation synthetic biology tools for use in streptomyces venezuelae. ACS Synth. Biol. 6, 159-166 (2017).

30. Bai, C. et al. Exploiting a precise design of universal synthetic modular regulatory elements to unlock the microbial natural products in Streptomyces. Proc. Natl. Acad. Sci. 112, 12181-12186 (2015). 
31. Jiang, F. \& Doudna, J. A. CRISPR-Cas9 Structures and Mechanisms. Annu. Rev. Biophys. 46, 505-529 (2017).

32. Boyle, E. A. et al. High-throughput biochemical profiling reveals sequence determinants of dCas9 off-target binding and unbinding. Proc. Natl. Acad. Sci. 114, 54615466 (2017).

33. Peters, J. M. et al. Enabling genetic analysis of diverse bacteria with MobileCRISPRi. Nat. Microbiol. 4, 244-250 (2019).

34. Lu, Z. et al. CRISPR-assisted multi-dimensional regulation for fine-tuning gene expression in Bacillus subtilis. Nucleic Acids Res. 47, e40-e40 (2019).

35. Dove, S. L. \& Hochschild, A. Conversion of the omega subunit of Escherichia coli RNA polymerase into a transcriptional activator or an activation target. Genes Dev. 12, 745-754 (1998).

36. Sudalaiyadum Perumal, A., Vishwakarma, R. K., Hu, Y., Morichaud, Z. \& Brodolin, K. RbpA relaxes promoter selectivity of M. tuberculosis RNA polymerase. Nucleic Acids Res. (2018) doi:10.1093/nar/gky714.

37. Guilinger, J. P., Thompson, D. B. \& Liu, D. R. Fusion of catalytically inactive Cas9 to Fokl nuclease improves the specificity of genome modification. Nat. Biotechnol. 32, 577-582 (2014).

38. Chen, S. P. \& Wang, H. H. An Engineered Cas-Transposon System for Programmable and Site-Directed DNA Transpositions. CRISPR J. 2, 376-394 (2019).

39. Xu, G. et al. 'Pseudo' gamma-butyrolactone receptors respond to antibiotic signals to coordinate antibiotic biosynthesis. J. Biol. Chem. 285, 27440-27448 (2010).

40. Wang, J. et al. A novel role of 'pseudo' $y$-butyrolactone receptors in controlling $\mathrm{Y}^{-}$ butyrolactone biosynthesis in Streptomyces. Mol. Microbiol. 82, 236-250 (2011).

41. Weber, T. et al. Metabolic engineering of antibiotic factories: new tools for antibiotic production in actinomycetes. Trends Biotechnol. 33, 15-26 (2015).

42. Kim, H. U., Charusanti, P., Lee, S. Y. \& Weber, T. Metabolic engineering with systems biology tools to optimize production of prokaryotic secondary metabolites. Nat. Prod. Rep. 33, 933-941 (2016).

43. Li, S. et al. Genome-Wide CRISPRi-Based Identification of Targets for Decoupling Growth from Production. ACS Synth. Biol. 9, 1030-1040 (2020). 
44. Bruder, M. R., Pyne, M. E., Moo-Young, M., Chung, D. A. \& Chou, C. P. Extending CRISPR-Cas9 Technology from Genome Editing to Transcriptional Engineering in the Genus Clostridium. Appl. Environ. Microbiol. 82, 6109-6119 (2016).

45. Huang, C.-H. et al. CRISPR interference (CRISPRi) for gene regulation and succinate production in cyanobacterium S. elongatus PCC 7942. Microb. Cell Factories 15, 196-196 (2016).

46. Wang, M., Liu, L., Fan, L. \& Tan, T. CRISPRi based system for enhancing 1butanol production in engineered Klebsiella pneumoniae. Process Biochem. 56, 139-146 (2017).

47. Cleto, S., Jensen, J. V., Wendisch, V. F. \& Lu, T. K. Corynebacterium glutamicum Metabolic Engineering with CRISPR Interference (CRISPRi). ACS Synth. Biol. 5, 375385 (2016).

48. Lv, L., Ren, Y.-L., Chen, J.-C., Wu, Q. \& Chen, G.-Q. Application of CRISPRi for prokaryotic metabolic engineering involving multiple genes, a case study: Controllable P(3HB-co-4HB) biosynthesis. Metab. Eng. 29, 160-168 (2015).

49. Kim, S. K. et al. CRISPR interference-guided balancing of a biosynthetic mevalonate pathway increases terpenoid production. Metab. Eng. 38, 228-240 (2016).

50. Batianis, C. et al. An expanded CRISPRi toolbox for tunable control of gene expression in Pseudomonas putida. Microb. Biotechnol. 13, 368-385 (2020).

51. Kampmann, M. CRISPRi and CRISPRa Screens in Mammalian Cells for Precision Biology and Medicine. ACS Chem. Biol. 13, 406-416 (2018).

52. Zhang, M. M. et al. CRISPR-Cas9 strategy for activation of silent Streptomyces biosynthetic gene clusters. Nat. Chem. Biol. 13, 607-609 (2017).

53. Wang, B., Guo, F., Dong, S.-H. \& Zhao, H. Activation of silent biosynthetic gene clusters using transcription factor decoys. Nat. Chem. Biol. 1-1 (2018) doi:10.1038/s41589-018-0187-0.

54. Enghiad, B. et al. Cas12a-assisted precise targeted cloning using in vivo Cre-lox recombination. Nat. Commun. 12, 1171 (2021).

55. Lee, N. et al. Thirty complete Streptomyces genome sequences for mining novel secondary metabolite biosynthetic gene clusters. Sci. Data 7, 55 (2020). 
56. Villegas Kcam, M. C., Tsong, A. J. \& Chappell, J. Uncovering the distinct properties of a bacterial type I-E CRISPR activation system. 2021.10.06.463193 https://www.biorxiv.org/content/10.1101/2021.10.06.463193v1 doi:10.1101/2021.10.06.463193.

57. Gibson, D. G. et al. Enzymatic assembly of DNA molecules up to several hundred kilobases. Nat. Methods 6, 343-345 (2009).

58. Engler, C., Kandzia, R. \& Marillonnet, S. A One Pot, One Step, Precision Cloning Method with High Throughput Capability. PLoS ONE 3, e3647 (2008).

59. Yu, X., Price, N. P. J., Evans, B. S. \& Metcalf, W. W. Purification and characterization of phosphonoglycans from Glycomyces sp. strain NRRL B-16210 and Stackebrandtia nassauensis NRRL B-16338. J. Bacteriol. 196, 1768-79 (2014).

60. Jakeman, D. L., Graham, C. L., Young, W. \& Vining, L. C. Culture conditions improving the production of jadomycin B. J. Ind. Microbiol. Biotechnol. 33, 767-772 (2006).

61. Martinez-Farina, C. F. et al. Isolation and synthetic diversification of jadomycin 4Amino-I-phenylalanine. J. Nat. Prod. 78, 1208-1214 (2015). 


\section{FIGURES}

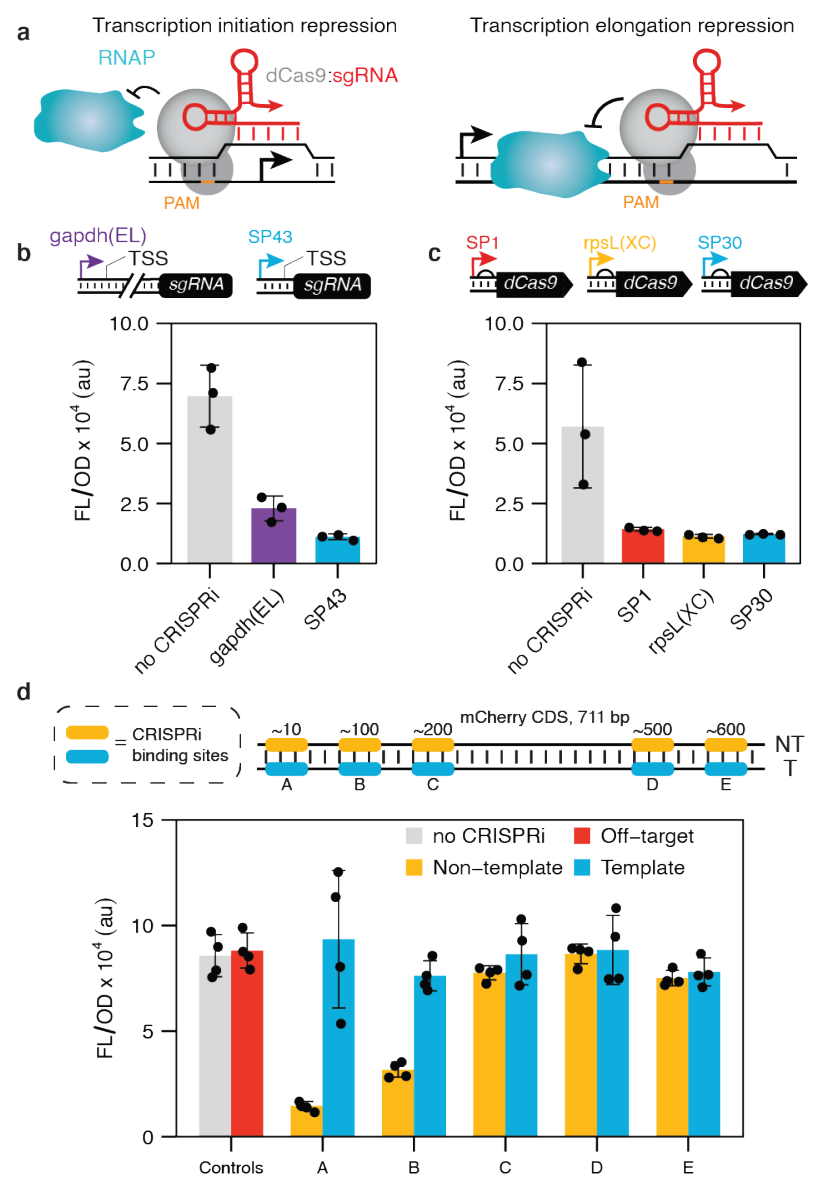

Figure 1. Creating a CRISPR interference (CRISPRi) system for Streptomyces venezuelae. a Schematic of CRISPRi mechanism. The ribonucleoprotein complex formed by dCas9 (colored grey) and the single guide RNA (sgRNA, colored red) binds to the promoter or gene coding region to block transcription initiation or elongation by RNA polymerase (RNAP). b Eliminating intervening nucleotides between the promoter and the sgRNA leads to higher repression by CRISPRi. Schematic of sgRNA expression cassette with the gapdh(EL) and SP43 promoter. The SP43 promoter contains an annotated TSS, whereas gapdh(EL) TSS remains unannotated. Fluorescence characterization of $S$. venezuelae cells conjugated with CRISPRi plasmid variants designed to repress transcription of a genomically integrated mCherry reporter. c. CRISPRi produces robust repression independently of dCas9 expression. Schematic of dCas9 expression cassette using the SP1, $\operatorname{rpsL}(\mathrm{XC})$, and SP30 promoters (ascending strength). Fluorescence characterization of $S$. venezuelae cells conjugated with CRISPRi plasmid variants designed to repress transcription of a genomically integrated mCherry reporter. d. 
Position-dependent repression by CRISPRi. Schematic of the sgRNA binding sites used that target different PAMs in the non-template (NT) and template (T) strand of the $m C h e r r y$ reporter gene. Fluorescence characterization of $S$. venezuelae cells conjugated with CRISPRi plasmid variants. Fluorescence characterization was performed by bulk fluorescence measurements (measured in units of fluorescence [FL]/optical density [OD] at $600 \mathrm{~nm}$ ). Data represent mean values and errors bars represent standard deviation of at least 3 biological replicates. 
a

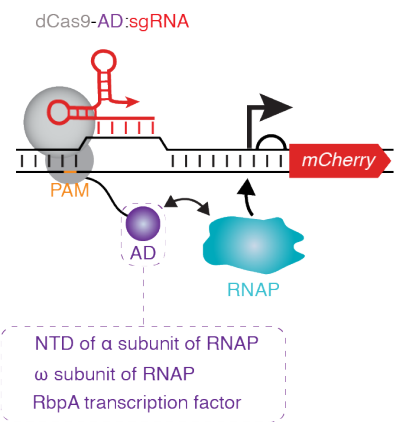

b Distance between PAM and TSS
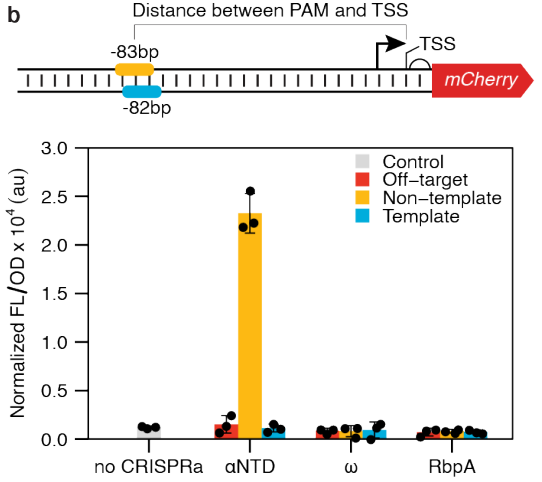

c Distance between PAM and TSS
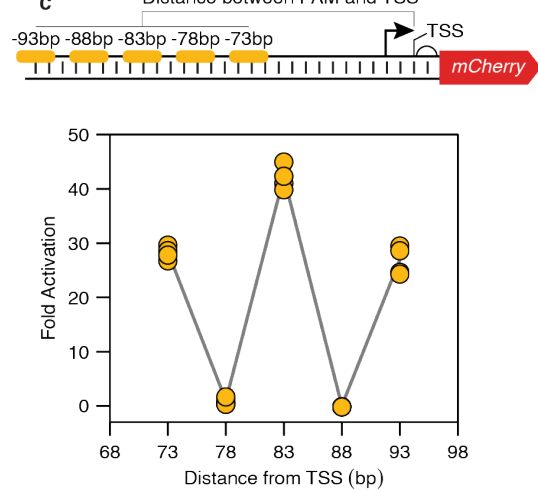

Figure 2. Creating a CRISPR activation (CRISPRa) for Streptomyces venezuelae. a Schematic of CRISPRa mechanism. An activator domain (AD, colored purple) is translationally fused to a dCas9 via a flexible linker. The CRISPRa complex binds upstream of a target promoter to recruit RNA Polymerase (RNAP) and activate transcription of the target gene. $\mathbf{b}$ The $\mathrm{N}$-terminal domain of the a subunit of RNAP ( $\alpha$ NTD) can serve as an AD for CRISPRa in Streptomyces. Schematic of sgRNA binding sites used that target the non-template (NT) and template (T) strand upstream of a promoter driving mCherry expression. The indicated distances reflect the number of nucleotides intervening between the 5' end of the PAM (not included) and the TSS (also not included). Fluorescence characterization of $S$. venezuelae cells conjugated with CRISPRa plasmid variants using different AD. c CRISPRa activation shows periodical distance-dependent activation patterns. Schematic of sgRNA binding sites used that target different sites on the non-template strand upstream of a promoter driving mCherry expression. Fluorescence characterization was performed by bulk fluorescence measurements (measured in units of fluorescence [FL]/optical density [OD] at $600 \mathrm{~nm}$ ). Fold activation was calculated by dividing the $[F L] /[O D]$ obtained in the presence of a CRISPRa against the no-CRISPRa control within each reporter plasmid. Data are reported as individual replicates with a line connecting the mean of each condition. 

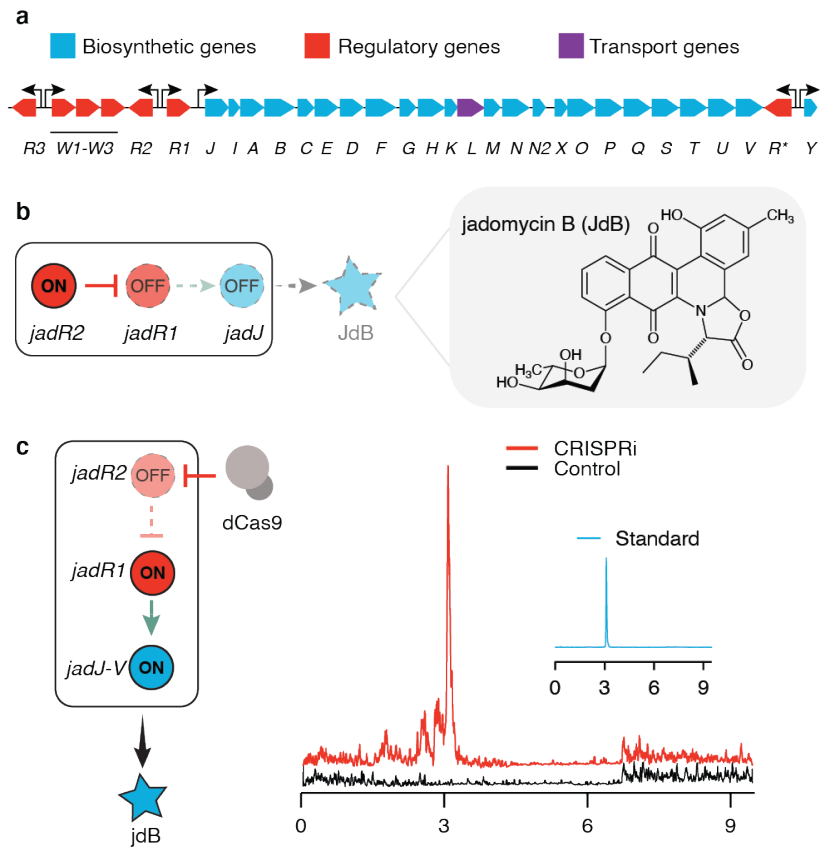

d
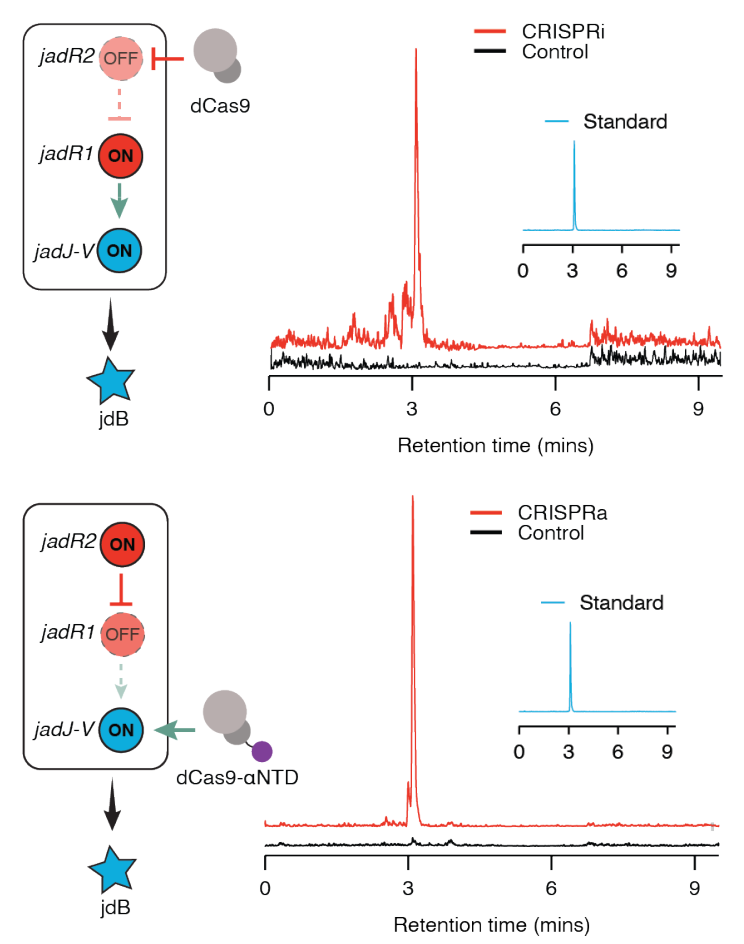

Figure 3. Using CRISPRi and CRISPRa to activate the silent jadomycin b (jdB) biosynthetic gene cluster (BGC). a Schematic of the jdB BGC of $S$. venezuelae. b Schematic of JadR1 and JadR2 regulation of the jdB BGC under normal laboratory conditions. Blunted arrows indicate repression and pointed arrows indicate activation. Dotted outline represents an inactive regulator and regulation step. Structure of jdB shown right of panel. c Schematic of CRISPRi repressing JadR1 to relieve repression on the jdB BGC and induce expression. LC-MS analysis of extracts from S. venezuelae conjugated with CRISPRi plasmids or no-CRISPRi control. Insert shows LC-MS analysis of a jdB standard. $d$. Schematic of CRISPRa activating the jadJ-V operon to induce expression of jdB BGC. LC-MS analysis of extracts from $S$. venezuelae conjugated with CRISPRa plasmids or no-CRISPRa control. Insert shows LC-MS analysis of a jdB 
bioRxiv preprint doi: https://doi.org/10.1101/2021.10.28.466254; this version posted October 28, 2021. The copyright holder for this preprint (which was not certified by peer review) is the author/funder, who has granted bioRxiv a license to display the preprint in perpetuity. It is made available under aCC-BY-NC 4.0 International license.

standard. Data in d and e are extracted ion chromatograms at $\mathrm{m} / \mathrm{z} 550.2059([\mathrm{M}+\mathrm{H}]+)$ of one representative biological replicate for each condition. Other biological replicates are shown in Supplementary figure 6. 